\title{
La Vierge sans âge. Mythes et rites, images et parentés
}

The ageless Virgin.Myths andrituals, images and kinships.

\section{Salvatore D'Onofrio}

\section{(2) OpenEdition}

1 Journals

Édition électronique

URL : http://journals.openedition.org/imagesrevues/1599

DOI : 10.4000/imagesrevues. 1599

ISSN : 1778-3801

\section{Éditeur :}

Centre d'Histoire et Théorie des Arts, Groupe d'Anthropologie Historique de l'Occident Médiéval, Laboratoire d'Anthropologie Sociale, UMR 8210 Anthropologie et Histoire des Mondes Antiques

\section{Référence électronique}

Salvatore D'Onofrio, "La Vierge sans âge. Mythes et rites, images et parentés », Images Re-vues [En ligne], 9 | 2011, mis en ligne le 25 novembre 2011, consulté le 30 janvier 2021. URL : http:// journals.openedition.org/imagesrevues/1599; DOI : https://doi.org/10.4000/imagesrevues.1599

Ce document a été généré automatiquement le 30 janvier 2021.

Images Re-vues est mise à disposition selon les termes de la Licence Creative Commons Attribution Pas d'Utilisation Commerciale 4.0 International. 


\title{
La Vierge sans âge. Mythes et rites, images et parentés ${ }^{1}$
}

The ageless Virgin.Myths andrituals, images and kinships.

\section{Salvatore D'Onofrio}

\author{
« Le créateur a enfanté le créateur, la servante a \\ enfanté le maître, la fille a enfanté le père ; fille \\ de sa nature divine, mère de sa nature \\ humaine "; « Fille de Dieu, mère de Dieu $»^{2}$.
}

\section{Le rôle des images}


1 Il n'y a guère longtemps que les images font l'objet d'une réflexion systématique en anthropologie. Carlo Severi ${ }^{3}$ les a associées à la parole chez les peuples sans écriture, Philippe Descola les a reconduites à la figuration, aspect constitutif des quatre formules ontologiques à travers lesquelles s'organise la perception du monde: l'animisme, le naturalisme, le totémisme

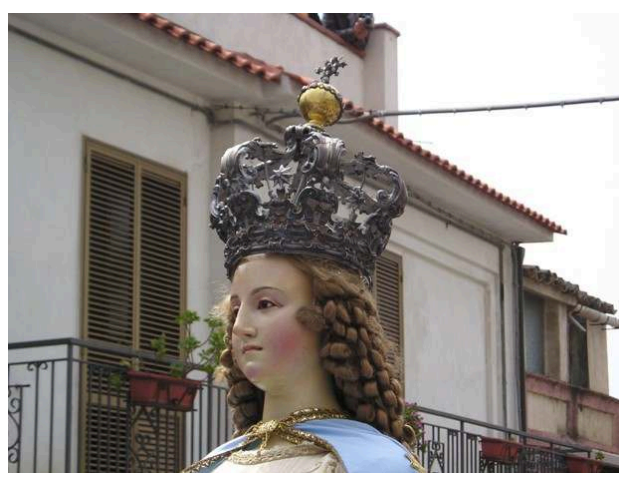
et l'analogisme. Chacun de ces modes d'identification réserve aux images un traitement différencié, ce qui permet de qualifier visuellement les existants auxquels on impute une intériorité et une physicalité semblable ou divergente de celles dont les humains font expérience.

Ces nouvelles perspectives obligent à prendre en compte plus que l'apport du génie individuel de l'artiste, les schémas conceptuels qui encadrent les images et leur confèrent un contenu social. Severi, en particulier, a proposé de prêter une plus grande attention au rôle de premier plan que celles-ci jouent dans les pratiques de mémorisation et d'action rituelle. D'où la notion de tradition iconographique destinée à intégrer celle de l'oralité.

Or, dans les sociétés de l'horizon chrétien, quelques ajustements sont nécessaires, car des traditions iconographiques fort différentes, mais reliées entre elles, ont servi à illustrer un "paquet mythique » dont la source principale demeure la Sainte écriture. C'est sans doute la parole qui, sous forme de lecture dominicale ou de sermons a maintenu vivante la "matière mythique " des Évangiles, mais avec un concours non secondaire des images: qu'elles soient celles des catacombes, de l'art cultivé de la Renaissance ou des rituels populaires contemporains. De toute façon, l'ensemble de ces traditions iconographiques et verbales est à l'origine des représentations mentales qui actualisent les mythes et qui sont le véritable objet de notre étude. À côté de chaque mot, la mémoire emmagasine des images, dans une relation très étroite - de terme à images, pourrait-on dire - ce qu'illustre la définition saussurienne du signifiant comme " image acoustique $»^{5}$. Le rapport interne au signifiant est néanmoins plus compliqué, puisqu'il est vrai qu'à chaque son correspond un large éventail d'images, mais il ne reste que l'usage concret de la langue en réduit sensiblement le nombre et les transforme en stéréotypes. Ce n'est pas par exemple à toutes les images de courage ou de table que l'on pense lors des actes de parole qui les évoquent, mais aux représentations qui permettent de faire économie de leur dimension iconographique et de ne retenir que leurs aspects les plus formels. Or ces représentations mentales, au contenu figuratif appauvri, sont celles-là mêmes dont l'esprit humain se sert pour actualiser les mythes.

\section{Mère et fille de son fils}

4 Tout en tenant compte du rapport des images à la parole, nous essayerons d'explorer davantage la manipulation des faits de parenté, notamment spirituelle, d'où elles résultent. 
5 À cette fin, je commencerai par un vers parmi les plus beaux de La Divine comédie, dans lequel Dante Alighieri affiche une des configurations parentales centrales du Christianisme: "Vierge mère, Fille de ton Fils " ${ }^{6}$. Dans la prière que Bernard de Clairvaux adresse à la Vierge, au début du dernier chant du Paradis, pour qu'elle accorde à Dante avec la vision de Dieu la révélation des mystères suprêmes, l'illustre poète exprime bien la complexité de la relation entre la mère et le fils racontée dans les Évangiles, complexité qui trouve son origine dans la conception virginale du Christ et dans la Trinité. De cette complexité témoignent d'autres œuvres littéraires, parmi lesquelles le premier quatrain du Morgante de Pulci : «Et toi, Vierge, fille et mère et épouse.... ».

Le paradoxe incarné par la filiation renversée et les vierges mères n'est pas exclusif de l'horizon chrétien. Dans la tradition chi'ite, par exemple, Fatima «la vierge », fille du Prophète, est la Umm Abîhâ, autrement dit la " Mère de son père ", et cela selon une tradition remontant à la poésie du mystique soufi Mansur al-Hallaj ${ }^{8}$. Plus généralement, comme l'a montré de manière exemplaire Lévi-Strauss par son analyse du vers d'Apollinaire «mères filles de leurs filles » dans le poème Les Colchiques, à l'origine de ces "figures de pensée " on trouve des "mystères théologiques progressivement laïcisés par la poésie savante, la littérature courtoise et le langage de naturalistes ${ }^{9}$.

7 Nous pouvons affirmer par ailleurs que les croyances et les configurations parentales sous-tendues à ces figures de pensée possèdent un caractère presque universel, comme le montrent les mythes des vierges mères étudiés par l'anthropologue italien Giuseppe Cocchiara ${ }^{10}$ et dans le fameux article de Leach Virgin Birth ${ }^{11}$. Tous les deux discutent de la maternité virginale en la rapprochant surtout du problème de la prétendue ignorance du processus physiologique de la paternité chez certains peuples primitifs ${ }^{12}$. Ce qui a été le moins étudié c'est le rôle joué par les images dans les pratiques religieuses qui accompagnent ces mythes ainsi que dans les relations de parenté symbolique qui articulent les échanges, de tout genre, entre les mondes divin et humain.

8 L'étude la plus complète du vers de Dante - «Vierge mère, fille de ton fils » - a été faite par Auerbach ${ }^{13}$, qui y a vu un exemple sublime de poésie chrétienne fondée sur les paradoxes de la foi : "Dieu et homme, créateur et créature, parole et chair, mort et résurrection, mère et vierge ». Auerbach remarque également une utilisation consciente du parallélisme antithétique de la rhétorique grecque et l'influence directe de l'interprétation figurative de la Bible.

9 Nous sommes redevables à Carlo Ossola d'avoir mis en lumière l'importance de l'iconographie dans cette affaire ${ }^{14}$. Il repère le principe générateur du vers de Dante dans la tradition théologique et figurative byzantine qui associe l'image de la «dormition » de la Vierge à celle de l'ascension au ciel de sa petite âme (l'animula) que le Christ tient dans ses bras - la mère de Dieu ne meurt pas, elle transite, ou bien elle quitte la vie de ce monde en s'endormant entourée par les apôtres. L'animula de la Vierge est en chair et en os. Suivant une tradition médiévale qui représente l'âme comme un enfant, la filiation renversée dont il est ici question semble trouver une expression picturale appropriée. Il n'empêche que dans quelques cas, dont la rareté n'est pas au détriment de sa valeur emblématique, c'est une jeune adolescente que son Fils tient sur ses genoux au-dessus de la scène de la dormitio Virginis ${ }^{15}$. Cette scène est souvent surmontée par une autre où la Vierge, destinée elle aussi à monter au ciel, a le même âge du Christ qui la couronne ou qui est assis tendrement à ses côtés. Par un 
curieux glissement sémantique, on appelle parfois cette dernière image de la Vierge "Assomption » (par exemple celle de Cimabue à Assisi), alors qu'à proprement parler on ne représente par ce terme que son ascension au ciel. Signalons également que dans quelques cas la Vierge est couronnée en même temps par Dieu et par le Christ.

Ces différents cas de figure de la Vierge - un personnage presque sans âge, pourrait-on dire, car elle peut en recouvrir plusieurs - sont d'autant plus remarquables que son Fils a toujours trente-trois ans, et qu'ils sont proposés verticalement suivant la progression de l'âge de la Vierge. C'est un "paquet iconographique» dont on ne peut pas traiter séparément les éléments qui le composent : ils sont tous justifiés par la même logique de manipulation symbolique de la parenté tracée dans la sainte écriture. Bien que très peu développée, l'image de l'animula en jeune fille témoigne, comme nous le verrons plus précisément par la suite, de l'alliance symbolique entre la mère et son fils à côté d'autres images représentant le renversement dans la filiation.

\section{La Dormition de la Vierge}

11 La «Dormition de la Vierge » n'est pas mentionnée dans les Evangiles canoniques. Dante a certainement pu s'inspirer des mosaïques absidales réalisées à la fin du XIII ${ }^{\mathrm{e}}$ siècle pour l'église de Sainte Marie Majeure à Rome par Jacopo Torriti (fig. 1-2), selon un modèle qui trouve l'une de ses meilleures expressions dans l'église palermitaine de Sainte Marie de l'Amiral du XII ${ }^{e}$ siècle (fig. 3-4) : la filiation renversée est représentée ici parfaitement, sur deux arcs en vis-à-vis, par la naissance du Christ dans les langes et la dormitio virginis. La Vierge, sa robe et son grabat sont les mêmes, mais une petite variation est visible dans le motif du lange emmaillotant les deux enfants, certainement pour signaler la différence de genre ${ }^{16}$.

Fig. 1

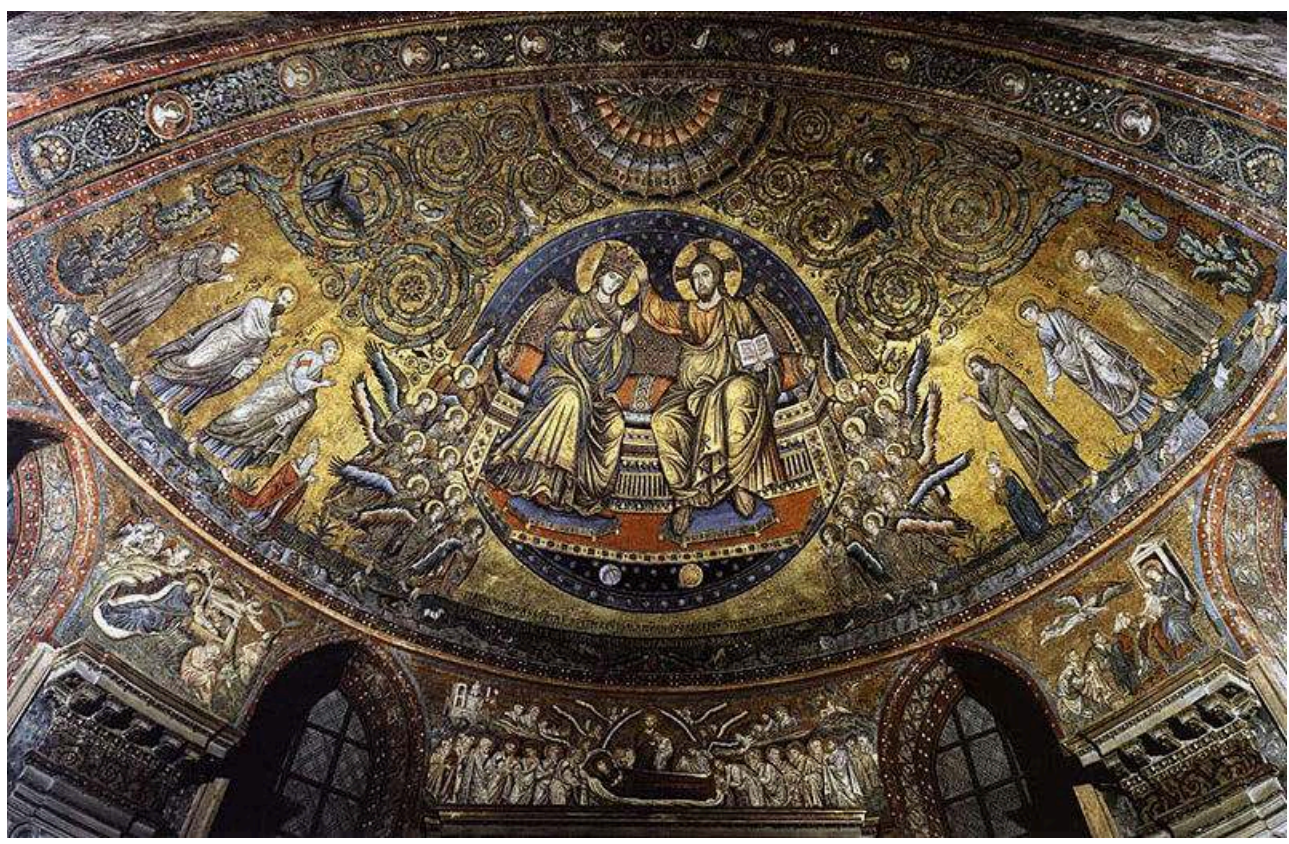

Jacopo Torriti, Rome, Basilique de Santa Maria Maggiore. Dans la partie inférieure, Dormitio virginis, en haut le Couronnement (1295). 
Fig. 2

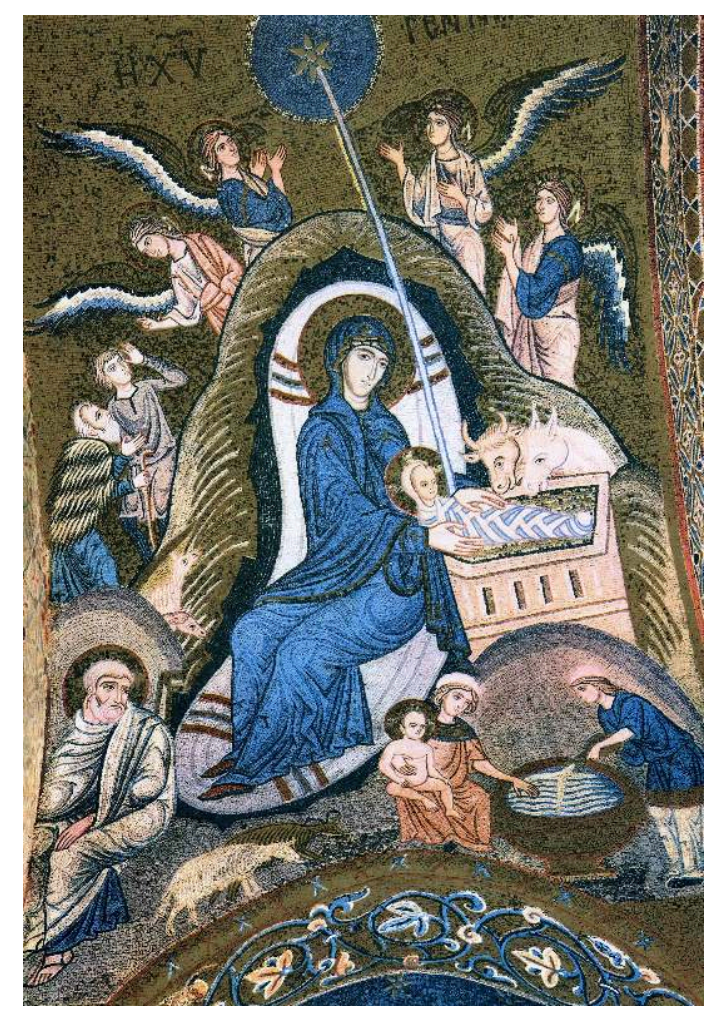

Palerme, Santa Maria dell'Ammiraglio, Nativité

Fig. 3

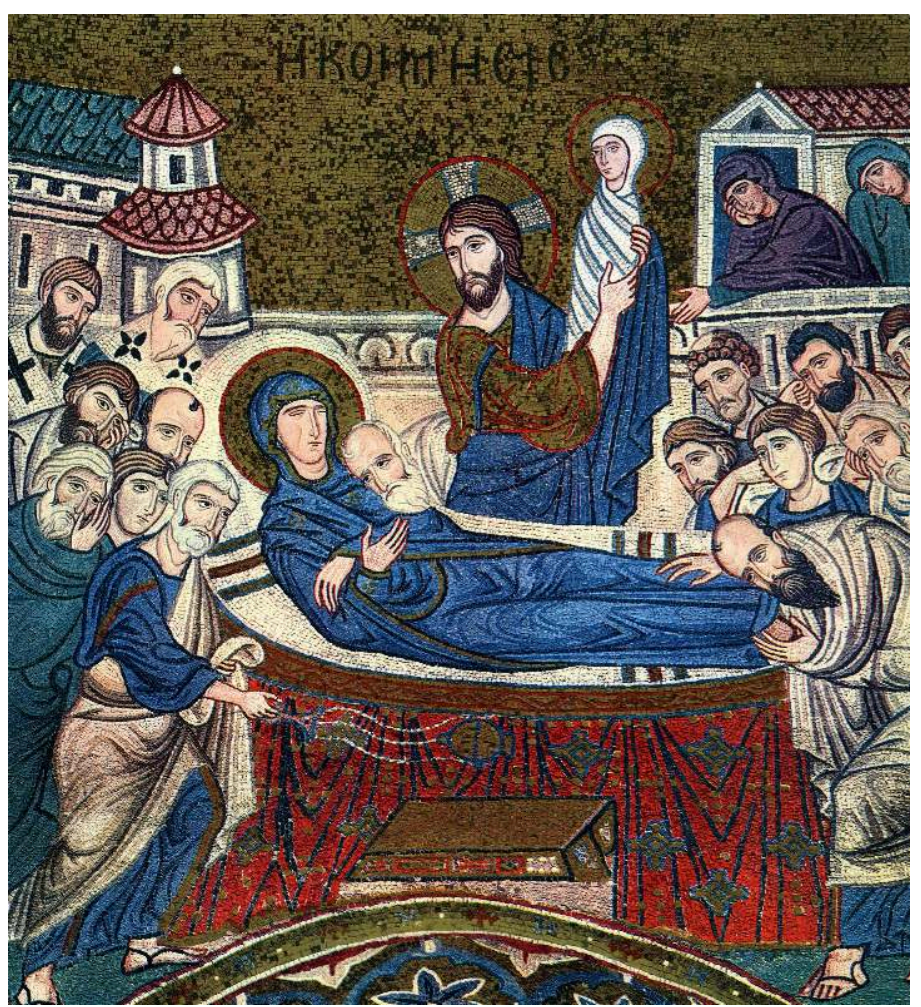

Palerme, Santa Maria dell'Ammiraglio, L'ascension de l'animula. 
12 Pour montrer la variété des âges de la Vierge, rappelons, parmi d'autres, une Dormitio de Giotto (fig. 4) qui se trouve au Musée de Berlin, une autre du XV siècle peinte pour une fresque de l'église romane de Troia (fig. 5) dans la province de Foggia, une Assomption de la même époque dans l'église de Santa Maria de Monteluce (fig. 6), à Pérouse. Comme dans d'autres cas, cette image de l'Assomption est proposée toute seule.

Fig. 4

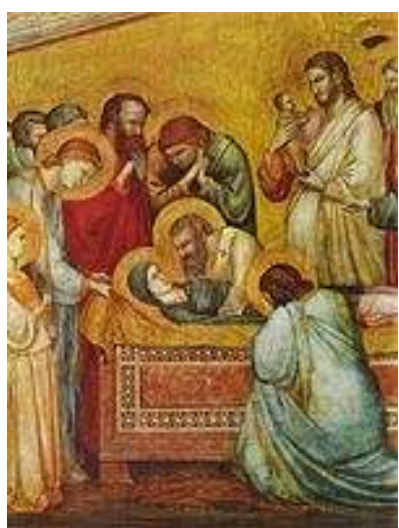

Giotto, Dormitio virginis, Berlin, Staatliche Museen, Gemaeldegalerie.

Fig. 5

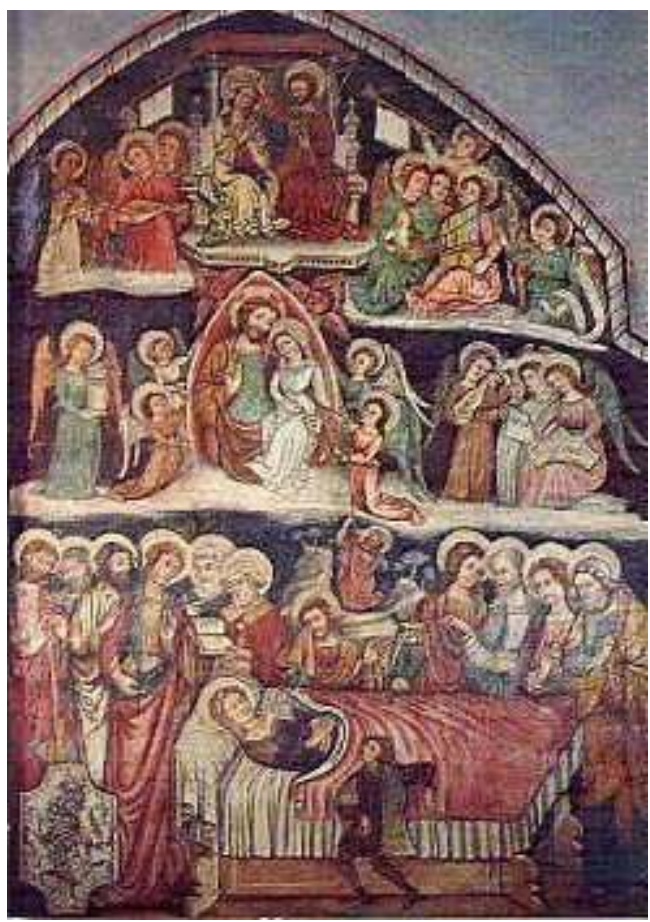

Foggia, Cathédrale de Troia, Dormitio virginis, Assomption et Couronnement. 
Fig. 6

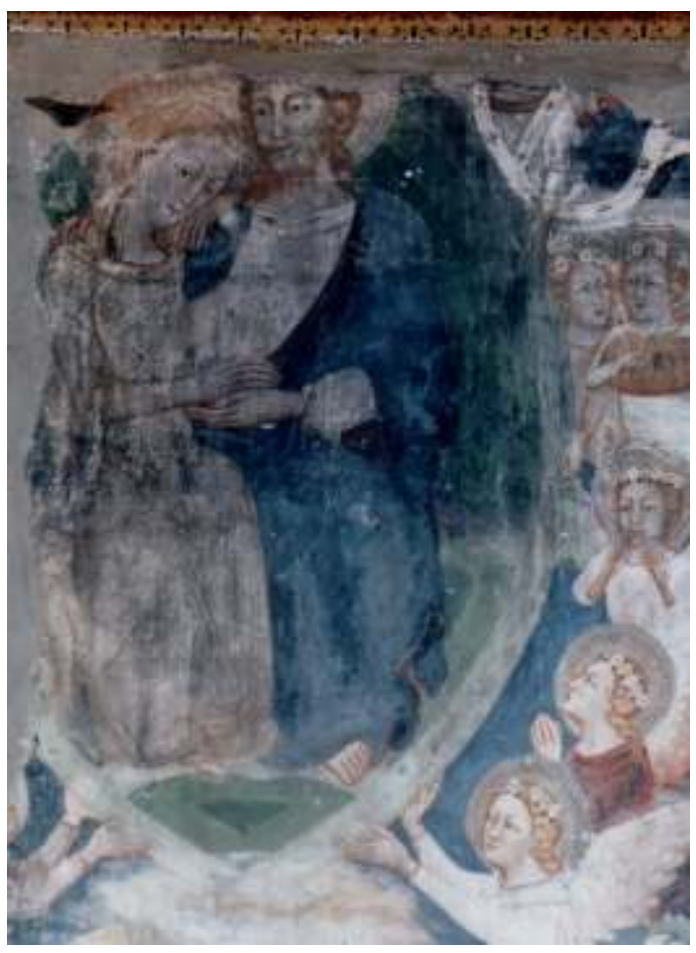

Pérouse, Chœur du Monastère de Monteluce, Assomption, début du xive siècle.

13 Nous sommes ici en présence de possibilités combinatoires permises par la tradition iconographique, mais façonnées également par l'ancrage des images à une parole écrite dont on ne saurait pas se passer. Le poète aussi ne semble pas échapper au «contrôle » exercé par l'écriture au moyen des images sacrées.

En effet, il est certain que Dante s'est inspiré de l'iconographie byzantine, car durant l'année 1300 il était à Rome à l'occasion du Jubilé, mais on ne peut pas douter qu'il connaissait aussi le débat théologique lancé sur ce thème dès le $\mathrm{IV}^{e}$ siècle et notamment dans les apocryphes réunis sous le titre de Dormitio Mariae Virginis ou Transitus Mariae Virginis $^{17}$. Dans ces apocryphes, on affirme la thèse du corps non corrompu de Marie après sa Dormition ou sa mort et de son immortalité à travers le déplacement de son âme et de son corps - au départ séparés - dans les hauts lieux de l'Ascension du Fils.

Non acquise par la résurrection, l'immortalité de Marie s'intègre au trait qui la caractérise davantage : sa maternité virginale. La réunion simultanée en une même femme de la double condition de vierge et de mère fait de Marie, du point de vue de la dimension temporelle, un personnage hors norme. Le corps non corrompu de la Mère de Dieu traduit la possibilité de prendre aux côtés de son Fils non seulement plusieurs âges mais aussi les statuts parentaux (et implicitement sexuels) qui leur correspondent. Les pères de l'Eglise et les théologiens ont souvent réfléchi sur ces " anomalies » des figures divines par rapport aux communsdes mortels, comme le témoigne de manière exemplaire ce qui écrit Jean Damascène au VIII ${ }^{\mathrm{e}}$ siècle : « il convenait que celle qui avait gardé intacte sa virginité dans l'enfantement, conservât son corps exempt de toute corruption après la mort. Il convenait que celle qui avait porté dans son sein, sous la forme d'un enfant, le Créateur, résidât dans la semence divine. Il convenait que l'Epouse de Dieu pénétrât dans la maison céleste. Il convenait que celle qui avait vu son Fils sur la Croix et reçu ainsi dans son cœur la douleur dont elle avait été exempte au 
moment de l'enfantement, contemplât ce Fils assis à la droite du Père. Il convenait que la Mère de Dieu possédât ce qui revient à son Fils, et qu'elle fût honorée comme Mère et Servante de Dieu par toutes les créatures ${ }^{18}$. Pie XII cite justement cette homélie de Saint Jean Damascène dans sa Constitution apostolique « Deiparae Virginis Mariae " pour justifier, en 1949, sa proposition de dogme de l'Assomption de la Vierge.

Comme nous l'avons dit, la tradition figurative de la Dormition, représente souvent, dans la partie supérieure, la scène du Couronnement de la mère par son fils, et parfois, dans la partie inférieure, la scène de l'ange du Seigneur qui coupe avec son épée de feu les mains du juif Jefonia au moment où il essaye de violer le cadavre de la Vierge. Cette dernière scène est illustrée elle aussi dans les apocryphes, où l'on raconte également des mains tranchées de Jefonia miraculeusement rattachées à ses bras après son repentir et sa conversion (fig. 7) ${ }^{19}$. On veut ainsi témoigner l'impossibilité de corrompre le corps de Marie, de son vivant ainsi qu'après sa mort.

Fig. 7

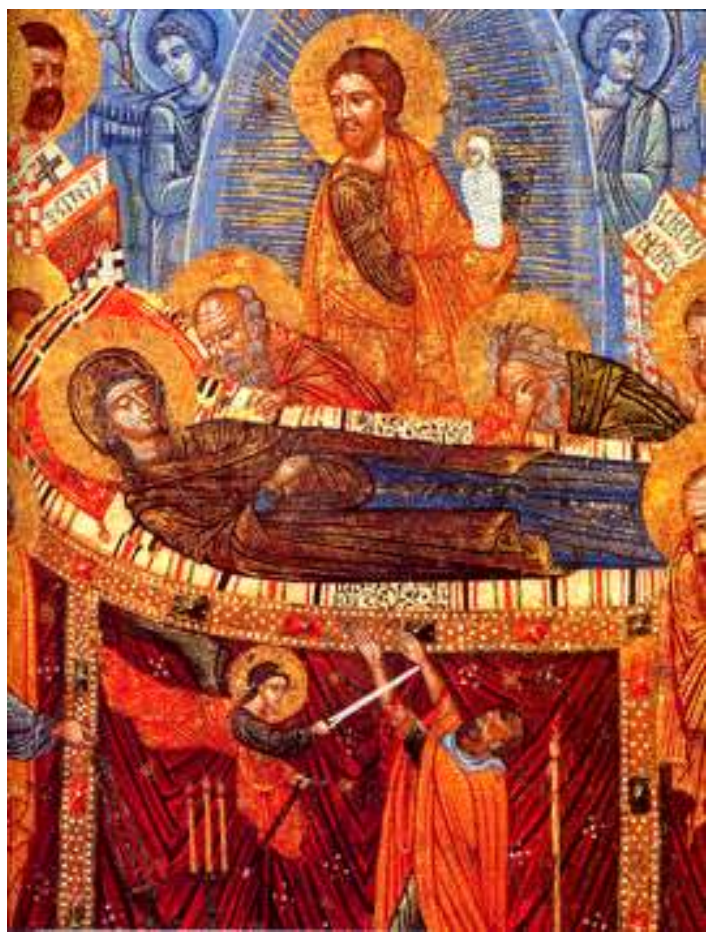

Icône de Onufri Qiprioti, Dormitio Virginis, fin XVIe siècle, Institut pour les Monuments culturels, Tirana.

Un symbole commun aux représentations que nous venons d'afficher (et qui lie strictement la mère et le fils) est enfin celui de la vesica piscis, la « vessie de poisson » ou "mandorle », qui résulte de l'intersection de deux cercles dont le centre de chacun fait partie de la circonférence de l'autre (fig. 8). Ce symbole a été différemment interprété par la tradition chrétienne : alors que le fruit ne représenterait que la nature divine du Christ cachée dans sa nature humaine (fig. 9), la grande auréole elliptique que l'on appelle « mandorle mystique », peut renfermer autant le Fils tout seul qu'avec sa mère ou sa mère avec lui ou bien les deux ensemble à la même âge. Par sa signification sexuelle, car elle rappelle jusqu'au geste contemporain des féministes formé par les pouces et les index des deux mains joints verticalement entre eux, la mandorle peut symboliser la gloire rayonnant de la personne divine du Christ en tant que verbe 
incarné ou bien le sexe féminin et la virginité de Marie, ou encore la croyance en une relation entre époux divins. Jean Clair a formulé récemment l'hypothèse que cette mandorle est " une matrice ", et, dans la symbologie médiévale, « l'accès à l'utérus qui renferme l'embryon $»^{20}$. A vrai dire, il n'y a pas de texte au Moyen Âge qui propose ce rapprochement ; ce n'est que la plaie du Christ qui est pensée par certaines mystiques comme une matrice. Il n'empêche qu'une série de "consonances iconographiques " centrées sur la sexualité et la hiérogamie symboliques mériteraient d'être développées dans cette direction. Ce symbole a été enfin adopté dans des pays du Nouveau Monde comme le Mexique où il encadre, entre autres, la Vierge de Guadalupe.

Fig. 8

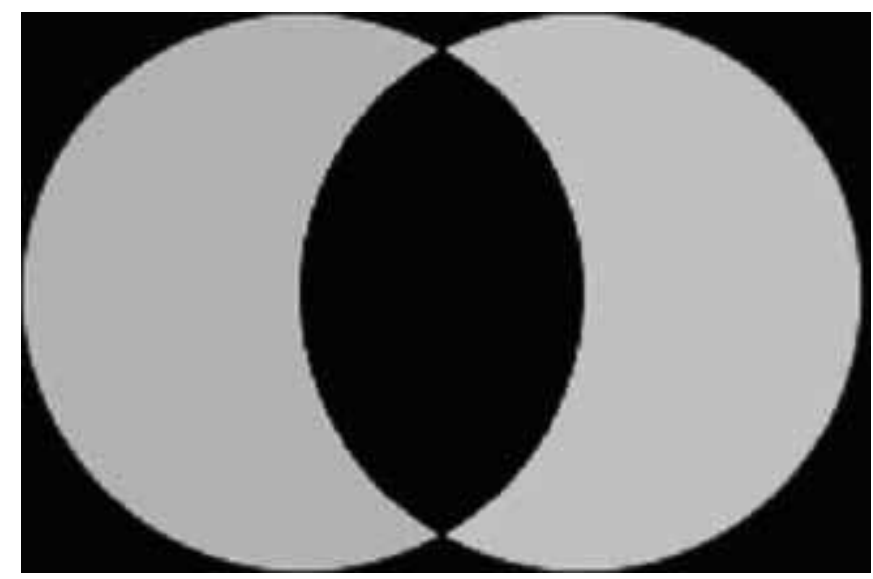

L'amande/Mandorle 
Fig. 9

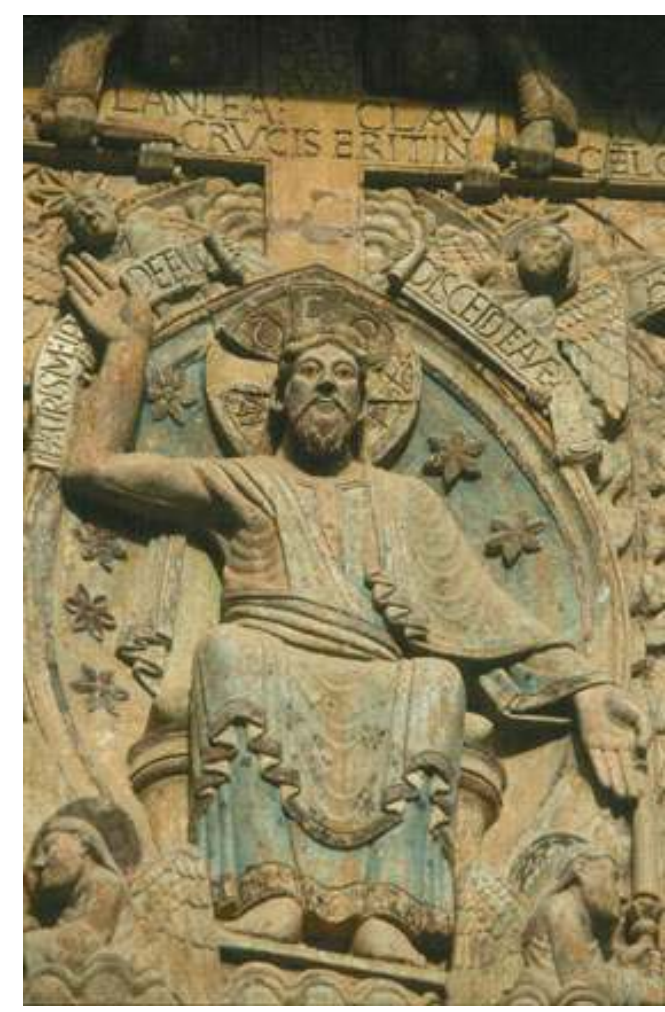

Le Christ dans une mandorle dans le tympan de la façade occidentale de l'abbatiale de Sainte Foy de Conques en Aveyron, XII siècle.

Se limiter à analyser les croyances, plus ou moins explicites, concernant la relation entre la Mère et son Fils, expose sans doute au risque de concevoir le sacré et les mythes qui lui correspondent, surtout dans le registre de la sexualité, comme des sphères autonomes de la praxis rituelle. En d'autres termes, il reviendrait à affirmer une relation hiérarchique entre le rite et le mythe. Également, ce n'est pas suffisant de montrer que "les classifications établies par les rites révèlent les grands principes structuraux d'organisation de la société comme et parfois mieux que les classifications explicitées par les mythes ${ }^{21} »$. Plus que de reconnaître l'efficacité symbolique du rite à coté de celle du mythe, il s'agit de construire le modèle de la relation que chaque société a élaboré entre eux. De ce point de vue, les positions théoriques de Lévi-Strauss sont emblématiques, puisqu'elles divergent des analyses concrètes qu'il a consacrées à ce rapport. Dans le «Finale" de L'Homme nu, où il aborde dans quelques pages très denses cette question, il propose non seulement d'étudier le rituel "en lui-même et pour lui-même, afin de comprendre en quoi il constitue un objet distinct de la mythologie", mais il conçoit également la relation entre le mythe et le rite comme une "opposition entre le monde vécu et le monde pensé" : "l'essence du rituel - souligne Lévi-Strauss - est de tenter de réduire la pensée à la vie ». Et pourtant, en dépit de cette fragilité de la réflexion lévistraussienne, l'analyse structurale, comme le montrent les articles contenus dans la section «mythologie et rituel» de Anthropologie structurale deux (ou ceux de la section "Croyances, mythes et rites » de Le regard éloigné), est sans doute la mieux placée pour comprendre comment et pourquoi le mythe et le rite s'entremêlent. Tous les articles réunis dans cette section n'apparaissent pas pertinents, mais «La geste d'Asdiwal »ou« Comment meurent les mythes » montrent clairement 
une des fonctions principales des rites : rendre efficace le principe de conservation de la matière mythique en dépit de toute transformation que le temps et la transmission dans l'espace infligent aux narrations. C'est à ce niveau que nous pouvons repérer le rôle des traditions iconographiques. Elles ne peuvent pas empêcher que les mythes se transforment - en traditions légendaires ou en récits visant à légitimer l'histoire - mais c'est bien grâce à elles et à leur rapport privilégié aux rituels que les formes se conservent et que les mythes persistent.

En passant aux pratiques religieuses, nous postulons ainsi que le rite n'est pas une simple déclinaison opérationnelle du mythe ni un "mouvement de la pensée », mais qu'il est nécessaire à sa reproduction, ce qui se fait au moyen de paroles proférées, gestes accomplis, objets manipulés, aussi bien qu'au moyen de bruits, de silences et surtout d'images.

\section{Le rajeunissement de la Vierge}

Les représentations savantes des liens entre la Vierge et le Fils - celle, poétique, de Dante ou bien celle, iconique, des artistes byzantins - trouvent une confirmation singulière dans un trait de la « culture figurative populaire » de l'horizon chrétien dont la valeur structurale expliquera nos choix de méthode relativement aux rapports entre le mythe et le rite, entre images et parenté.

21 Partout dans le Sud de l'Italie le Vendredi saint (Jeudi dans les villages de culte orthodoxe) a lieu la procession du Christ mort derrière lequel, immanquablement, nous trouvons la Vierge Douloureuse. Dans quelques endroits, le Dimanche de Pâques, est aussi le jour de la rencontre entre le Christ ressuscité et sa Mère. Le rituel de la rencontre se retrouve aujourd'hui dans plusieurs pays de culte chrétien: de l'Italie à l'Espagne, du Brésil au Mexique.

Ce rituel s'accorde de manière sui generis au mythe, dans ces successives élaborations, en mettant en scène les rapports de parenté et en les manipulant du point de vue symbolique.

Par deux fois la Mère repousse, incrédule, la nouvelle de la résurrection du Fils que divers messagers lui apportent (l'Archange Michel, les Apôtres Pierre et Paul, Jean l'Evangéliste et d'autres encore); de manière canonique, à la troisième tentative, finalement, elle y croit ou bien décide d'aller voir. Nous disons «de manière canonique " parce que cette triple répétition, mise en exergue il y a presque un siècle par le folkloriste danois Axel Olrik ${ }^{22}$, est une des charpentes des fables, du mythe, mais aussi du rite.

Les statues du Christ et de la Vierge bougent donc l'une par rapport à l'autre, à partir de points scéniquement bien choisis, pour se conjoindre généralement face à l'Eglise mère. Cette scène, répétée parfois dans d'autres endroits, est parmi les plus émouvantes du calendrier catholique populaire. La Vierge est habillée, comme pour le Vendredi saint, d'une veste de couleur noire ou bleu obscure qui signale sa condition de Douloureuse ; au moment de la rencontre on la laisse tomber et une autre apparait de couleur claire, céleste ou blanc-bleu mais aussi verte ou rouge. En même temps les colombes sont libérées et les cloches, « liées » jusqu'à ce moment, se délient pour la fête afin de signaler la bonne nouvelle. Les deux statues sont souvent pourvues de mécanismes qui leur permettent, encore trois fois - souvent sous un ou deux arcs 
joliment décorés - de s'embrasser, de se faire des révérences, ainsi que d'autres gestes de reconnaissance, avant d'entrer ensemble dans l'église en ondoyant dans la commotion générale sur la foule. La révérence, et surtout le baiser prennent une valence originelle dans la ville sicilienne de Modique où la Vierge (qui ouvre ses bras) est appelée vasa-vasa, bise-bise (fig. 10). Ailleurs c'est le Christ qui embrasse les pieds de sa mère ou celle-ci qui embrasse le côté de son fils. Le sens de ce geste est en partie expliqué par le fait qu'à ce moment on échange un signe de paix pour mettre fin aux discordes entre amis ou dans la famille.

Fig. 10

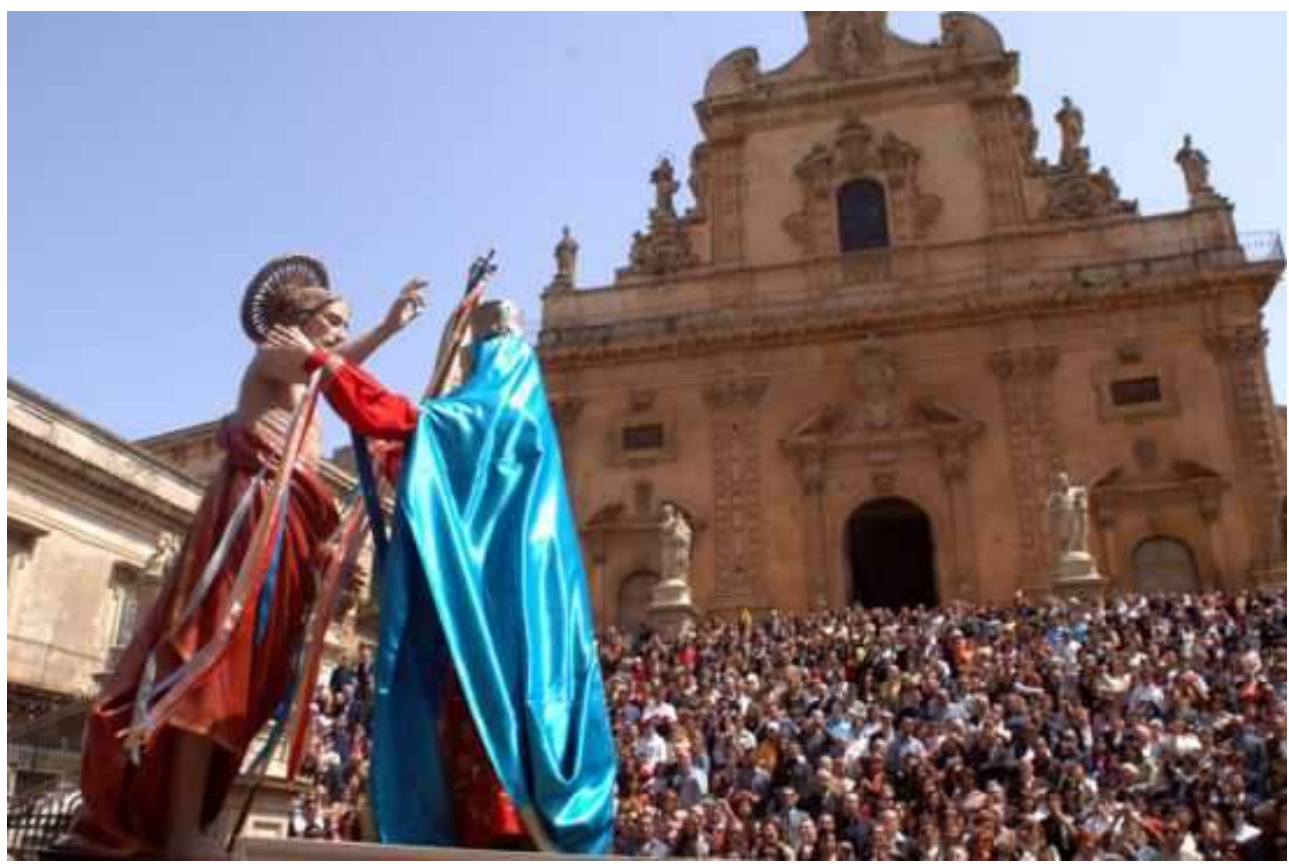

Modica, Rencontre du Ressuscité et de la Vierge bise-bise.

Nous sommes encore une fois en présence d'un ajustement structurel du texte évangélique où, avec les Apôtres, après la résurrection, plusieurs femmes figurent qui s'appellent Marie (plus une Jeanne et une Salomé). Marie Magdalena, dont l'évangéliste Jean raconte le dialogue avec le Christ ( femme, pourquoi pleures-tu... ») $)^{23}$, est toujours présente. De Marie, mère du Christ, aucune trace. Son corps disparaît, pourrait-on dire. 
Fig. 11

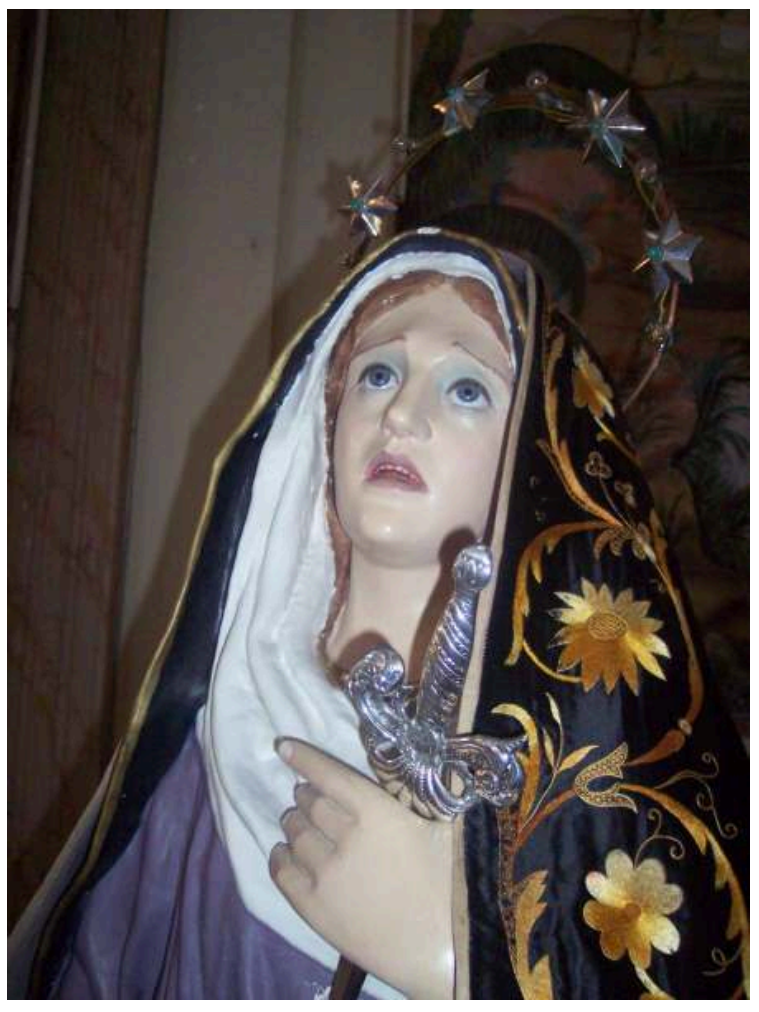

Aragona, Sicile, Vendredi saint, La Douloureuse.

Fig. 12

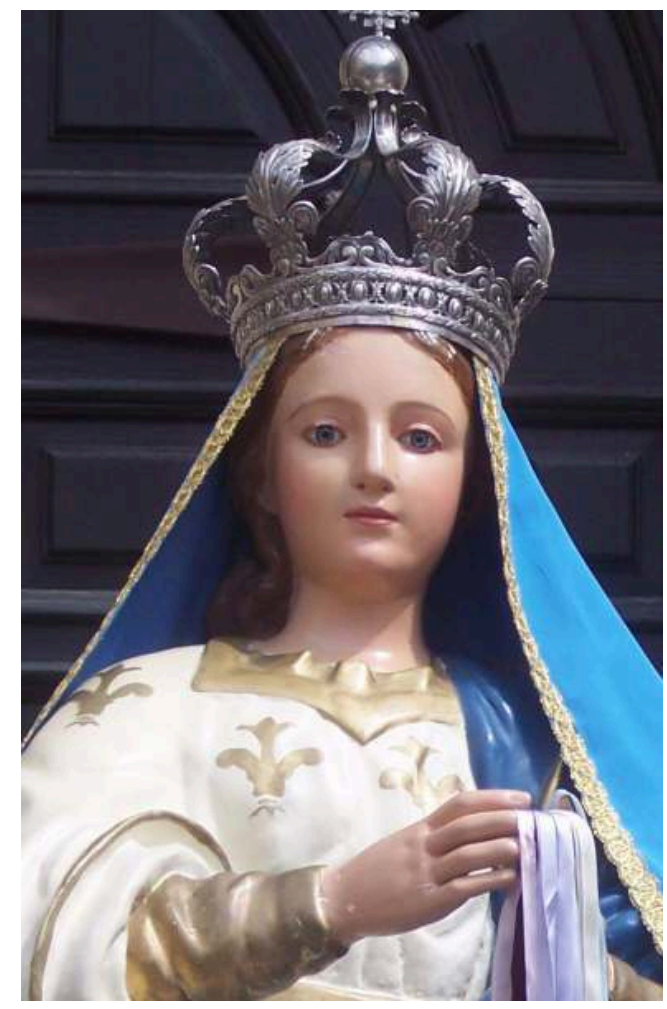

Aragona, Sicile, La Vierge du Dimanche de la Résurrection. 
Le détail le plus intéressant des rencontres de Pâques, tellement évident qu'il n'a pas été observé jusqu'ici, concerne néanmoins la statue de la Vierge. Il ne s'agit plus de la Mater Dolorosa, de 45-50 ans environ, qui, le Vendredi saint, suit le fils mort, recouverte de noir et avec le cœur transpercé par une ou plusieurs courtes épées. Dans la statue du dimanche, la Vierge Mère a non seulement une expression joyeuse, mais elle apparaît sensiblement rajeunie, du même âge sinon plus jeune que son fils. Quelques exemples seront ici suffisants, des fêtes de Pâques en Italie du Sud et en Espagne : Aragona (fig. 11-12) en Sicile et Villanueva de la Serena en Estremadura (fig. 13-14). Parfois, comme à Roccella Ionica en Calabre, la Vierge qui fait la révérence avec son fils ressuscité (la ncrinata $)^{24}$ non seulement rajeunit mais a des boucles, presque toujours de couleur blonde (fig. 15), ce qui serait impensable sur la tête de la Douloureuse, et même des pommettes rouges. Dans les cas, très rares, dans lesquels on utilise une seule statue ou bien où les deux Vierges ont plus ou moins le même âge, celui-ci est toujours très proche de celui du fils. Signalons que dans quelques petites villes siciliennes, les jeunes époux étaient obligés dans leur première année de mariage d'assister à la « rencontre ${ }^{25}$, ce qui témoigne d'une fonction propitiatoire liée à la fécondité que le couple divin est censé inconsciemment représenter.

Fig. 13

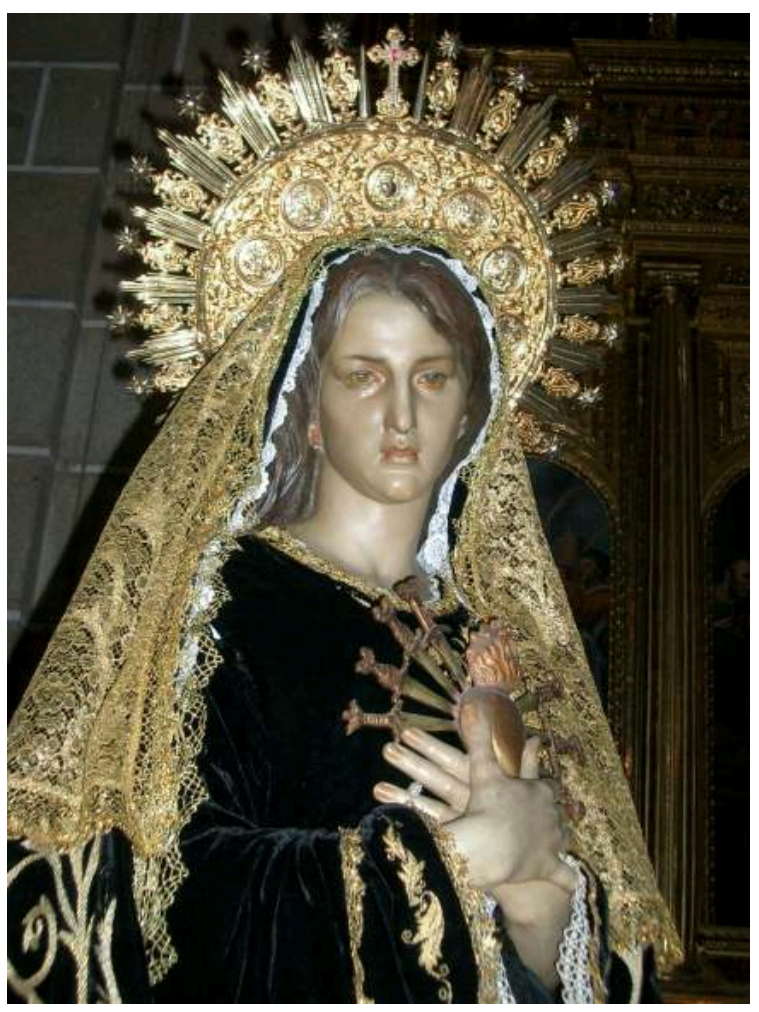

Villanueva de la Serena, Espagne, La Vierge des douleurs. 
Fig. 14

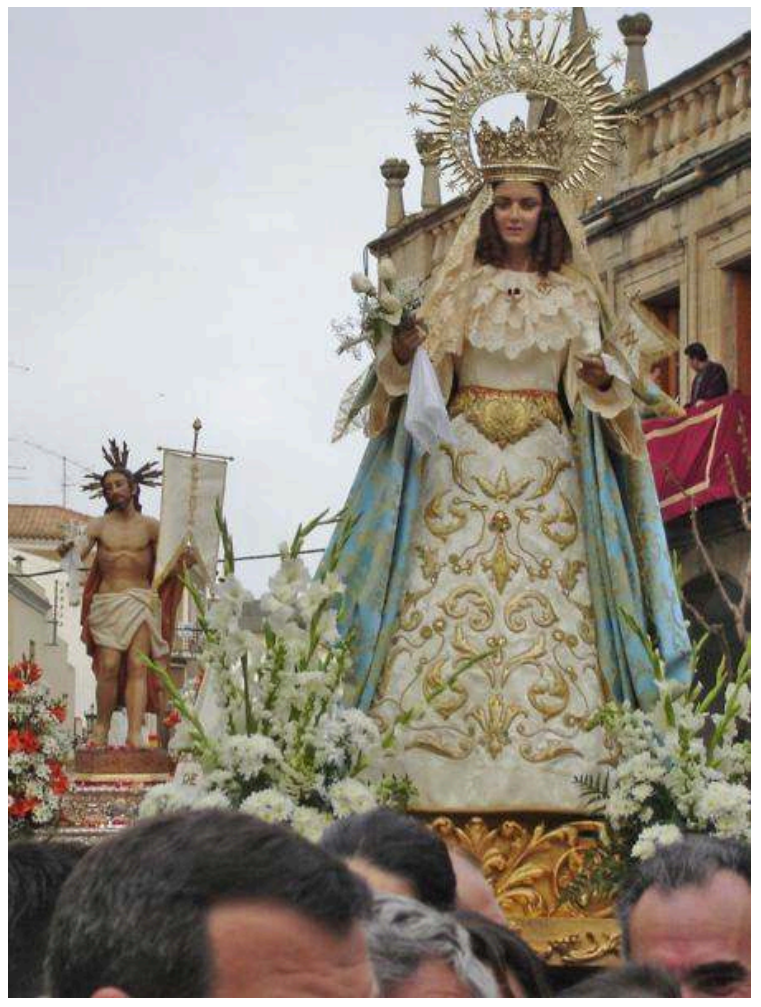

Villanueva de la Serena, Espagne, La Vierge de l'Aurore ou Carrerita.

Fig. 15

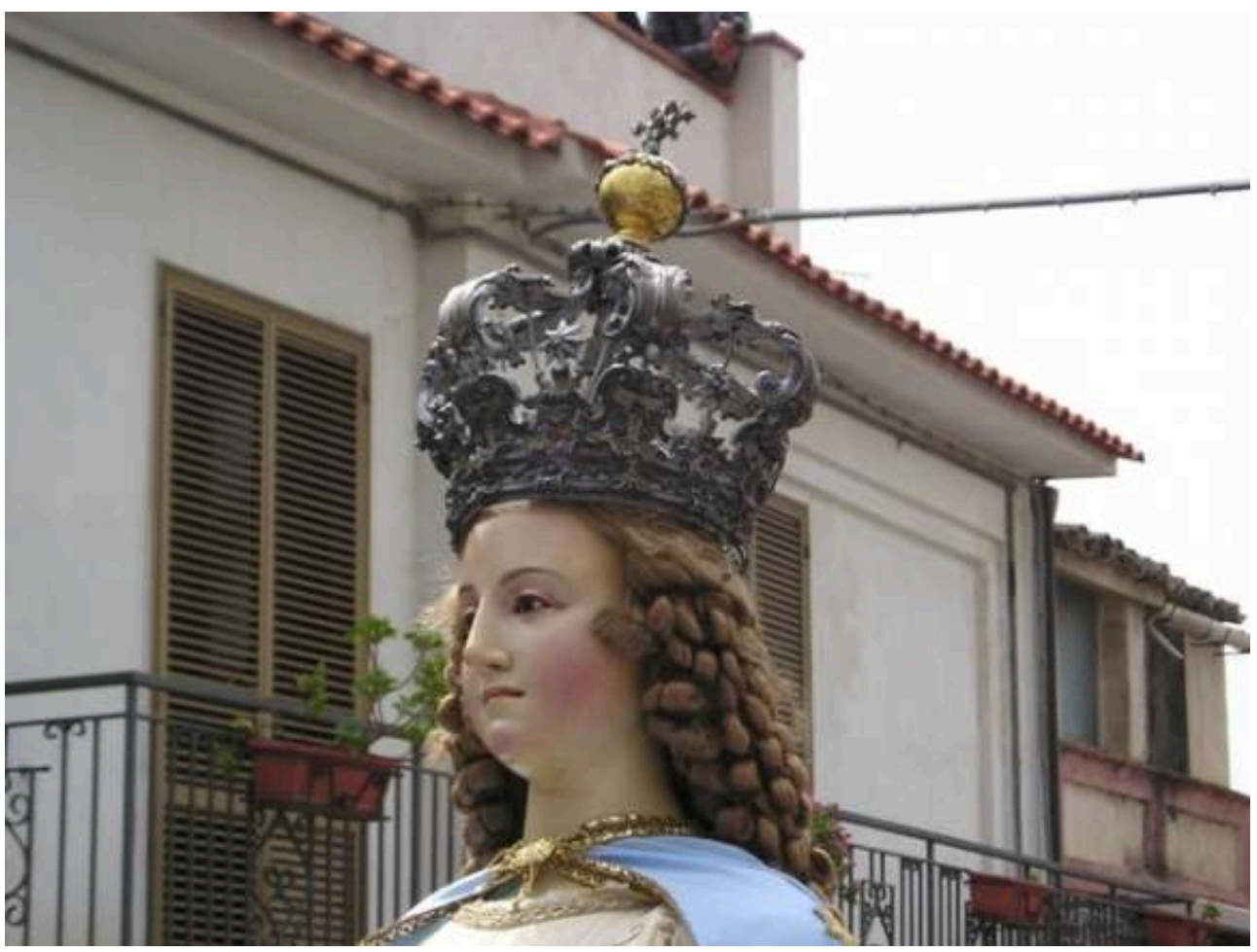

Roccella Ionica (Calabre), La Vierge aux boucles blondes et aux pommettes rouges. 

rencontres de Pâques, l'oralité se réduit parfois aux chants ou aux seules invocations hyperboliques adressées à la Vierge pour magnifier sa beauté - « vive la Vierge, vive le Ressuscité » ou bien "guapa», un peu partout en Espagne. C'est le corps des simulacres qui s'impose : en sollicitant le code des émotions, les mouvements induits par les porteurs visent à "animer» la configuration parentale qui est au coeur du christianisme. De ce point de vue, les simulacres de la Vierge et du Christ ressuscité apparaissent comme des véritables " artéfacts de la mémoire ", réactualisant, à chaque fois qu'on les met en scène, les représentations mentales qui leurs sont associées. À Villanueva de la Serena où la Vierge du dimanche prend le nom de Carrerita, dans la salle du banquet matrimonial, il est devenu d'usage ces dernières années de faire asseoir l'époux et l'épouse sur deux chaises l'un en vis à vis de l'autre et de les faire " courir ", "sautiller » et " rencontrer » exactement comme on fait avec les statue du Christ ressuscité et de sa mère. Il s'agit d'une interprétation populaire très pertinente du rituel sacré. Le caractère périodique de ces rituels - et des visites aux églises où les simulacres sont conservés - contribue à stabiliser les valeurs affichées par les figures divines : celles des liens de parenté tout d'abord. La tradition transcrit le mythe sur les statues, et ce sont ces icônes aux formes multiples qui s'intègrent, chez les couches populaires non alphabétisées, à la liturgie de la parole.

Un autre exemple emblématique du rajeunissement rituel de la Vierge vient d'une autre ville sicilienne, Petralia Soprana, la plus haute de la région montagneuse des Madonies, dans la province de Palerme. Ici, les statues de la Mère et du Fils ressuscité se rencontrent dimanche midi sur le parvis de la Cathédrale, où ils arrivent séparément et en partant à deux moments différents de la même église de Saint Salvatore qui les accueille pendant toute l'année. La Mère ne reconnaît pas le Fils en faisant deux fois un pas en arrière avant de se lancer la troisième fois vers lui. Ils «se font la bise» (si vàsanu), comme disent les gens, et, après une courte procession, ils rentrent, ensemble cette fois-ci, vers leur église mais pour aussitôt se séparer. La statue du Christ reprend sa place dans la sacristie, celle de la Mère à coté de l'enfant Jésus et de Saint Joseph dans le groupe statuaire de la sainte famille dont elle fait partie - il s'agit de trois statues en papier mâché réalisées au début du XXe siècle par les Malecore, artisans de Lecce, dans les Pouilles. Pour la «rencontre », on utilise donc une statue de la Vierge qui fait partie d'une Sainte Famille, autrement dit d'une femme à peine plus âgée qu'une adolescente.

En effet, plusieurs statues semblent représenter la Vierge à l'âge de la conception miraculeuse de Jésus, entre 15 et 16 ans selon les précisions apportées par quelques apocryphes. D'un point de vue symbolique, cela revient à mettre le Fils à la place du Père, non seulement le saint esprit qui l'engendra mais aussi Joseph, père « selon la chair ", dont l'importance est souligné par la généalogie : il appartient à la famille de David, ce qui conduit à Abraham d'après Matthieu (1,1-16), et même jusqu'à Dieu d'après Luc $(3,23-28)^{26}$. La rencontre entre le Christ et sa mère rajeunie justifie ainsi structurellement une des multiples configurations parentales auxquelles leur rapport donne lieu, aussi bien que l'absence de Joseph dans le complexe mythico-rituel concernant la mort et la résurrection du Christ. Joseph, comme Marie, dans les Évangiles canoniques ne meurt pas. La dernière fois qu'on le mentionne c'est au moment du pèlerinage à Jérusalem lorsque Jésus, âgé de 12 ans, était resté parmi les docteurs du temple sans en avertir ses parents. Les Apocryphes remédient aussi à cette

Images Re-vues, 9 | 2011 
incohérence, en faisant mourir Joseph le menuisier et en le faisant pleurer par la Vierge et le Christ.

L'« union sacrée » entre la Vierge et le Ressuscité est soulignée également par la présence d'apparats floraux (et corbeilles de fruits) qui parfois bougent eux aussi avec les simulacres, par l'explosion de pétards et le lancements de confettis, mais surtout par la course des deux simulacres ou de celui de la Vierge seule vers le Christ. Cette course peut être ouverte par des bandes d'enfants ou d'adultes (l'un d'entre eux a souvent une petite cloche à la main) qui peuvent également suivre les simulacres. Il arrive encore que les porteurs fassent sautiller les deux statues, surtout celle de la Vierge, ce qui produit une atmosphère d'excitation à laquelle tout le monde participe et qui rejoint son acmé au moment du geste symbolisant la rencontre. L'« union sacrée » peut enfin être soulignée par la présence de figures diaboliques ${ }^{27}$ qui essayent de gêner la rencontre ou par l'« ostension d'éléments végétaux » comme le basilic dont la valeur «fécondante » est largement attestée dans les poèmes enfantins et dans les chants populaires siciliens. ${ }^{28}$

Dans notre figuration statuaire, résultant d'une "théologie populaire inconsciente " que les artisans du papier mâché ou du gypse ont fait leur, la mère participe, de manière miraculeuse, de la régénération de la nature et du temps qui caractérise l'événement pascal, et ceci en accord avec la manipulation symbolique dont le rapport mère-fils est l'objet dans l'histoire sacrée des Évangiles. N'oublions pas, en effet, que dans la tradition catholique, la hiérogamie symbolique se réalise dans l'identification de Marie à l'Eglise épouse du Christ et que chez beaucoup de peuples de la Méditerranée la fécondation de la nature et du temps prévoit l'intervention rituelle d'un couple divin, souvent incestueux. Certes, il est vrai que dans le récit évangélique il n'est pas question de relation sexuelle entre la Mère et son Fils ni de transgression d'interdits puisque rien n'est dit à ce propos ${ }^{29}$. Il n'empêche que les figures de la mèreépouse ainsi que l'inversion dans la filiation ou l'élaboration de la parenté spirituelle relèvent de la nature symbolique des liens entre la Mère et son Fils: la maternité virginale et la trinité les placent en deçà de la Règle de la prohibition de l'inceste en justifiant toute manipulation et surtout celles qui ont lieu sur le plan sexuel. Le christianisme suit, de ce point de vue, une logique qui n'est pas différente de celles d'autres sociétés qui représentent le passage de la Nature à la Culture par un parcours à rebours - dans les mythes, les rites ou dans des formes concrètes d'organisation sociale $^{30}$. L'inceste et le conflit comme éléments fondateurs se rencontrent en plusieurs endroits, par exemple à l'occasion des fêtes de Pâques dans les régions occidentales et du Nord-Ouest du Mexique ${ }^{31}$, pendant le Carnaval dans les communautés Cora de la Sierra de Nayar ${ }^{32}$. On raconte ici que le Christ séduit la Vierge qui ne le reconnaît pas et, lors de la Semaine Sainte, le premier est tué par les Juifs (ses frères) qui vengent ainsi l'honneur de leur mère. Il s'agit du même "contenu fantasmatique incestueux » que Jérôme Baschet repère dans la tradition médiévale ${ }^{33}$.

32 Les manipulations symboliques permises dans le cadre rituel éclaircissent le sens profond des mythes évangéliques qui leurs correspondent. Ce sont les images standardisées par les rituels populaires ou par l'art cultivé qui stabilisent ces mythes en s'intégrant à leur lecture ou à leur écoute. Pour rester dans l'enceinte de notre sujet, rappelons qu'en dépit de son évolution artistico-rituelle, dans la tradition populaire ainsi que dans la peinture ou la statuaire, la représentation de la sainte famille s'accorde, même inconsciemment, avec l'idéologie sous-tendue au mythe évangélique. 
Mis à part la naissance, jusqu'à une certaine époque Jésus, Joseph et Marie n'étaient jamais représentés sans compter avec d'autres personnages, jusqu'à 23, parmi lesquels le petit saint Jean était toujours compris ; ce n'est qu'à partir du XV siècle que la sainte Famille fait son apparition toute seule en des moments différents de la vie du Christ. Il est intéressant de remarquer que les images canoniques prévoient également la représentation de Marie qui tient dans ses bras ou allaite son fils, de Joseph qui le tient lui aussi dans ses bras ou par la main, de saint Joseph tout seul ou bien de Marie toute seule dans des circonstances différentes, mais on ne voit presque jamais Joseph et Marie tous seuls ensemble. Ce couple, symboliquement stérile, n'existe qu'en fonction de la conception miraculeuse du Fils, ce qui renvoie à la configuration de l'atome de parenté spirituelle que nous avons mis en route.

\section{La parenté spirituelle}

Croyances sexuelles et pratiques religieuses explorées jusqu'ici représentent en effet un aspect central de la manipulation des rapports mère-fils au sein de ce que nous avons appelé l'atome de parenté spirituelle.

Ce dernier est le système de relation que nous avons postulé ${ }^{34}$ en développant la théorie de Lévi-Strauss du passage de la nature à la culture à travers la prohibition de l'inceste et l'exogamie. Notre atome de parenté spirituelle oppose dans l'horizon chrétien le couple mari-femme au couple compère-commère, et symétriquement au couple pèrefils le couple parrain-filleul, où le parrain prend la place qui est de l'oncle maternel dans l'atome de parenté lévistraussien ${ }^{35}$. Ce système a imposé une réflexion sur le rapport entre structure et histoire ou, pour être plus précis, sur le rapport entre les structures de l'histoire sacrée et les formes cultuelles et parentales qui, au cours du temps, surtout au moyen des représentations iconographiques, ont été appelées à les actualiser. En d'autres termes, le problème est de savoir comment les structures mythiques se convertissent en histoire à travers les images et les rites, qui montrent ainsi leurs fonctions incontournables.

Les Évangiles ne présentent aucune trace des relations et des termes de la parenté spirituelle ni des prohibitions matrimoniales qui leur sont associées, ce qui a induit les anthropologues fonctionnalistes à n'en rechercher l'origine que dans l'histoire des conciles ou dans les rapports avec le judaïsme et les cultures des sociétés non chrétiennes. L'étude structurale de la parenté spirituelle nous a permis, en revanche, non seulement d'en repérer le modèle fondateur précisément dans les Évangiles (et notamment dans la conception virginale du Christ par le Saint Esprit et dans la paternité sociale de Joseph) mais d'isoler (après Lévi-Strauss et Françoise Héritier) une autre modalité d'inceste, du troisième type, entre des individus qui ne sont pas forcément des consanguins ni des alliés. La prohibition de rapports sexuels entre compère et commère (c'est-à-dire entre le parrain d'un enfant et la mère de ce dernier) n'est qu'un résultat des possibles incestes du troisième type dans les différentes sociétés humaines.

Pour répondre à l'exigence, sans conteste universelle, de pourvoir l'enfant d'une paternité sociale, dans l'horizon chrétien la famille biologique père-mère-enfant, se dédouble en une famille symbolique (fig. 16). Cette famille symbolique est composée de la mère-commère, récupérant comme Marie à l'égard du père spirituel de son enfant une virginité inviolable; du fils-filleul, qui renaît comme le Christ en dehors de toute 
constriction biologique; du parrain-compère qui en assure à travers le baptême sa nouvelle ou deuxième naissance - comme l'Eglise elle-même l'appelle - en cumulant des fonctions qui dans le récit évangélique reviennent au Saint Esprit et à Joseph.

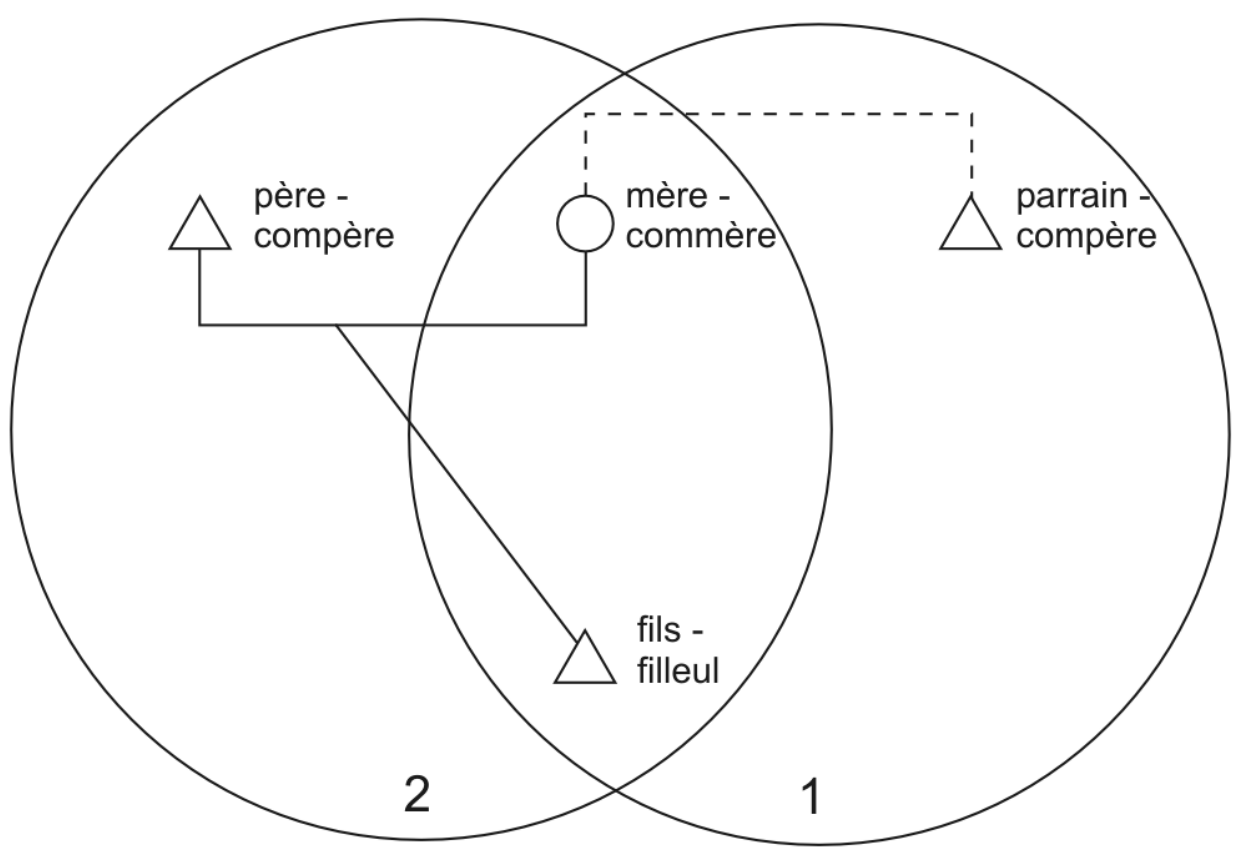

Famille biologique (2) et famille symbolique (1) réunies dans l'atome de parenté spirituelle. tirer des certitudes même dans les époques pré-sociales que les hommes imaginent par un parcours à rebours et dont les évidences physiologiques ont imposé, y compris dans un contexte spirituellement si marqué comme le christianisme, une référence indirecte à l'allaitement au sein du fils de la part de Marie dans un des Évangiles : "Comme Jésus était en train de parler, une femme éleva la voix au milieu de la foule pour lui dire : "Heureuse la mère qui t'a porté dans ses entrailles, et qui t'a nourri de son lait !" » (Lc 11, 27). La famille symbolique, dont les rapports se désexualisent sur le modèle de la Sainte Famille, s'avère ainsi comme nécessaire pour fonder la famille biologique ${ }^{36}$. La position de l'Église est, à ce propos, ambiguë, puisque elle considère la famille naturelle comme étant à la base de la construction du social d'où elle la fait résulter. En effet, pour l'Eglise, la famille biologique n'a d'existence qu'après le baptême d'un enfant et grâce à la présence d'un père spirituel : aux commencements le prêtre, puis un patrinus, pater divinus ou petit-père selon les différentes interprétations des théologiens qui toutes nous conviennent.

En rapprochant des relations entre la Vierge et son Fils la « spiritualisation de la chair» et l' " engendrement sans semence » qui composent à travers le baptême l'atome de parenté spirituelle, on peut saisir un mécanisme d'inversion très subtil entre les pratiques religieuses rituelles et les croyances sexuelles mythiques.

Ce que le mythe autorise (et s'autorise) sur l'axe de la filiation divine n'est possible que parce qu'il est strictement interdit dans la réalité sociale. Bien que la prohibition frappe 
manifestement le couple de parents symboliques formé par la mère et par le père spirituel de l'enfant, au niveau profond elle vise également le couple conjugal, dont la relation sexuelle ne devrait être finalisée, selon l'Eglise, qu'à la procréation. En d'autres termes: d'un côté le travail symbolique légitime la réalité des relations de type biologique en leur imposant un modèle mythique a-sexualisé, de l'autre la force du biologique et de la sexualité se déploie dans cette dimension symbolique en multipliant de manière sui generis tous ses recours.

En explorant la nature des relations entre la Vierge et son Fils, dans le mythe et dans le cadre rituel, nous avons ainsi pu comprendre, à travers les images, non seulement que ceux-ci sont inséparables et s'ajustent en permanence au sein d'un univers limité de possibles, mais que les phénomènes par lesquels on les met en œuvre - les croyances, les pratiques, la religion et le sexe - font l'objet d'une manipulation symbolique dont l'étude demeure en anthropologie incontournable.

\section{NOTES}

1. Cet article reprend un chapitre du livre Le sauvage et son double, paru en octobre 2011 aux éditions Les Belles Lettres dans la collection « Vérité des mythes » dirigée par Bernard Deforge.

2. PSEUdo-Augustin, Sermo de Virginitate Mariae (Patrologia latina, Supplément, II, col. 1187) ; Sermo 195,

3 (ibid.,39, col. 2108). Cit. in C. LÉVI-STRAUSS, « Une petite énigme mythico-littéraire ", in Le regard éloigné, Paris, Plon, 1983, p. 294-5).

3. C. SEVERI, Le principe de la Chimère. Une anthropologie de la mémoire, Paris, Rue d'Ulm/Musée du Quai Branly, 2007.

4. Ph. DESCOLA, Par-delà nature et culture, Paris, Gallimard, 2006.

5. F. DE SAUSSURE, Cours de linguistique générale, Paris, Payot, 1995.

6. DANTE ALIGHIERI, La Divine Comédie : Le Paradis/Paradiso, trad. fr. J. Risset, Paris, Flammarion, 2004, ch. XXXIII, 1.

7. L. PULCI, Morgante, Milano, Mondadori, 1994, ch. I, 2(1).

8. Cf. L. MASSIGNON, Akhbar Al-Hallaj, recueil d'oraisons et d'exhortations du martyr mystique de l'Islam, Paris, Vrin, 1975.

9. C. LÉVI-STRAUSS, « Une petite énigme mythico-littéraire », in Le regard éloigné, op. cit., p. 296.

10. G. COCCHIARA, « "Come si nasce". Sopravvivenze delle credenze primitive sulla maternità nelle tradizioni popolari ", Lares, vol. XI, p. 269-275; id., "Come si nasce. Le vergini-madri nella novellistica popolare e nella storia delle religioni ", in Il paese di Cuccagna e altri studi di folklore, Turin, Boringhieri, 1965, p. 13-53.

11. E. LEACH, «Virgin Birth ", in Proceedings of the Royal Anthropological Institute of Great Britain and Irelandfor1966, 1969, p. 39-50 [rééd. in Genesis as Myth and Other Essays, London, Jonathan Cape, 1969: 85-112, n., p. 117-122; trad. fr., "Les vierges-mères ", in L'unité de l'homme et autres essais, Paris, Gallimard, p. 77-107].

12. Cf. S. D'ONOFRIO, "La vierge et le marié lié », in L'Esprit de la parenté, préf. F. Héritier, Paris, Éditions de la Maison des sciences de l'homme, 2004.

13. E. AUERBACH, Écrits sur Dante, trad. fr. D. Meur, Paris, Macula, 1999. 
14. Et cela bien qu'il rappelle que dans la Lettra a Cangrande, Dante Alighieri (Epistola a Cangrande, ed. E. Cecchini, Florence, Giunti, 2005), en s'appuyant sur l'autorité d'Aristote, considère son texte «complètement vrai dans la lettre avant que dans l'allégorie» (C. ossola, Dante nel Novecento Europeo, www.ladante.it/culturaitaliana. 2006). À vrai dire, dans cette Epistola, dont la paternité se discute encore, Dante insiste sur une définition polysémique de son oeuvre et qualifie sa signification allégorique comme profonde par rapport à sa signification littérale. Rappelons, d'autre part, que l'interprétation médiévale, fondée sur «le sens quadruple de l'écriture » (littéral, allégorique, tropologique et anagogique), ne peut pas renoncer à la distinction primaire entre signification littérale et signification figurée.

15. Nous trouvons également plusieurs exemples où la Vierge en prière est âgée de 5 à 8 ans et le Christ la tient débout contre lui : par exemple l'enluminure « La mort de Marie » de Jean Fouquet (tiré du Livre d'Heures d'Etienne Chevalier) et la tempera «La mort de la Vierge » de Duccio di Buoninsegna (Duomo di Siena).

16. Il serait intéressant de pouvoir établir la datation précise de cette mosaïque, car l'église date du 1143, mais elle a été détruite et restaurée plusieurs fois. La dormitio virginis ne devient populaire qu'après la diffusion au XIII ${ }^{e}$ siècle de la Légende Dorée de Jacques de Voragine. Après le Concile de Trente, la Dormition de la Vierge se transforme généralement en Assomption.

17. Sur le contexte hébraïque de ce traité cf. F. MANNS, Le récit de la dormition de Marie (Vat. Grec 1982). Contribution à l'étude des origines de l'exégèse chrétienne, Jerusalem, Franciscan Printing Press, p. 121-144, cit. in K. SCHREINER (VERGINE, MADRE, REGINA. I volti di Maria nell'universo cristiano, trad. it., Rome, Donzelli, 1995, p. 54 ss.) qui relève les «symboles convergents » de la " palme », de la « nuée », de la « lampe » et du « parfum ».

18. Homilía II in dormitionem B. V. Mariae, 14 [PG 96, 742].

19. Écrits apocryphes chrétiens, Paris, Gallimard, 1997, p. 165-188. Dans le Transitus, l'antagoniste juif, lui aussi repenti, s'appelle Ruben.

20. J. CLAIR, «Deuil et féminité: l'iconographie de la Pietà dans la tradition chrétienne », in L. Anvar et alii, Les femmes, l'amour et le sacré, Paris, Albin Michel, 2010, p. 18.

21. J. CUISENIER, Penser le rituel, Paris, PUF, 2006, p. 24.

22. A. OLRIK, "La costruzione del racconto : le leggi epiche ", introd. et trad. it. de J. VIBAEK, in Uomo e cultura, VI (11-12), 1973, p. 197-232.

23. Jean 20, 11-13, La Bible de Jérusalem, Paris, Les Éditions du Cerf, 1998.

24. Un autre terme significatif de cette rencontre est en Calabre le « dévoilement » (la sbilata).

25. Nous l'avions remarqué dans un article sur les symboles des fêtes de Pâques : S. D'ONOFRIO, Le parole delle cose. Simboli e riti sociali in Sicilia, Galatina, Congedo, 1997 [Pubblicazioni del Dipartimento di Filologia, Linguistica e Letteratura dell'Università di Lecce, 12], p. 178.

26. P.-E. DAUzat («Mythologie de l'engendrement et du sexe chez les Pères de l'Église », in Diogène, 208, 2004, p. 16-29) a attiré l'attention sur les paradoxes de la «naissance virginale » à partir de la dissonance de l'Évangile de Luc par rapport à celui de Matthieu. Le problème est à vrai dire plus compliqué, puisque dans l'Évangile selon Matthieu (1, 18-25), Joseph, après avoir pris chez lui sa femme enceinte, comme l'Ange du Seigneur lui avait prescrit en songe, « il ne la connut pas jusqu'au jour où elle enfanta un fils, et il l'appela du nom de Jésus ». L'Evangile n'exclut donc pas qu'il l'ait connue après la naissance de Jésus.

27. Cf. I. ButTITTA, «L'abballu di li diavuli e lu ncontru a Prizzi », in Le feste di Pasqua, rédaction de S. D'Onofrio, Palerme, Sicilian tourist service, 1990, p. 169-174. La présence de diables est attestée aussi dans quelques communautés mexicaines.

28. I. BUTTITTA, Verità e menzogna dei simboli, Roma, Meltemi, 2008.

29. Cf. J. BASCHET, Le sein du père. Abraham et la paternité dans l'Occident médiéval, Paris, Gallimard, 2000, p. 52 ss. 
30. Ce que nous proposons par les exemples de l'organisation familiale des $\mathrm{Na}$ de Chine et le fameux rituel de Nouvelle Guinée, le Naven des Iatmul du Moyen Sépik où le travestissement d'un oncle maternel en sœur et la simulation d'un acte sexuel avec le neveu utérin représente la situation de promiscuité primitive précédant l'état social (cf. S. D'ONOFRIO, L'esprit de la parenté, Europe et horizon chrétien, préf. de Françoise Héritier, Paris, Maison des sciences de l'homme, 2004, p. 19-22).

31. Cf. C. BONFiglioli, A. GUTIÉRREZ et M. E. OlAVARRÍA, « De la violencia mítica al 'mundo flor'. Transformaciones de la Semana Santa en el norte de México", Journal de la Société des Américanistes, 90, 1, 2004, p. 57-91. L'idée de fertilité liée à la rencontre entre le Ressuscité et sa Mère, est corroborée dans l'horizon chrétien par d'autres traditions. Dans quelques communautés du Nord du Mexique, à Nouvelle Laredo par exemple, il existe la tradition, d'origine anglo-saxonne et arrivée des États-Unis, des œufs cachés par le lapin de Pâques. Le dimanche de Pâques, dans ces endroits, prend ainsi le nom de jour de la lapine. En dépit de l'origine nord-américaine de la fête, le lapin est lié, dans quelques cultures amérindiennes, à d'autres formes zoologiques, comme le tatou, qui l'intègrent du point de vue linguistique et symbolique. Ce dernier est aussi lié à une géométrie symbolique permettant des rapprochements très intéressants avec la mandorle mystique.

32. Cf. P. ALCOCER, P. La Pachitas en Chuisete'e, information de terrain, ms. inédit., 1999 ; J. NEURATH, Hacia el Carnaval permanente. Tiempo, biopoder y transgresion ritual, Mexico, MNA, sous presse, 2011. 33. Cf. J. BASCHET, Le sein du père... op. cit, p. 56 et ID. La civilisation féodale, Paris, Aubier, 2004, p. 676-679.

34. S. D'ONOFRIO, L'Esprit de la parenté, op. cit.

35. Ce rôle de l'oncle maternel était largement présent non seulement dans le monde romain d'où vient le terme latin avunculus - mais jusqu'au Moyen-Âge, comme le montre la littérature épique.

36. Cf. S. D'ONOFRIO, L'Esprit de la parenté, op. cit.

\section{RÉSUMÉS}

Cet article étudie le statut des images concernant la configuration parentale la plus importante de l'horizon chrétien : le rapport entre la Vierge et son Fils. La tradition iconographique, surtout byzantine, a illustré les différentes facettes de ce rapport, comme celles qui sont engendrées par les croyances en la maternité virginale et en la Trinité. Elle a également contribué à stabiliser les mythes évangéliques (canoniques et apocryphes) qui l'ont mis en place. Strictement mêlées à la parole liturgique et aux rites populaires, les images permettent ainsi de mieux comprendre le travail symbolique sous-jacent à la filiation renversée (visible par exemple dans la Dormitio virginis). La hiérogamie, quant à elle, est représentée par de nombreuses images de l'Assomption et par des rencontres entre les statues de Pâques du Christ ressuscité et de sa mère rajeunie.

This article studies the status of images concerning the most important parental configuration of the Christian horizon: the relationship between the Virgin and her Son. The iconographic tradition, especially Byzantine, illustrated the different facets of this relationship, including those generated by the belief in the virgin birth and the Trinity. It also helped to stabilize the myths of the gospels (canonical and apocryphal) which promoted it. Strictly mixed with the liturgical word and popular rituals, the images help to understand better the symbolic work that 
underlies reversed filiation (visible for example in the Dormitio virginis). The sacred marriage is depicted in numerous images of the Assumption and in meetings between the Easter statues of the Risen Christ and his rejuvenated mother.

\section{INDEX}

Keywords : Anthropology, Christianity, Italy, kinship, Middle Ages, spiritual kinship

Mots-clés : Anthropologie, Christianisme, Italie, Moyen Âge, parenté, parenté spirituelle

\section{AUTEUR}

\section{SALVATORE D'ONOFRIO}

Salvatore D'Onofrio est anthropologue. Il effectue un travail de terrain en Sicile et dans les Pouilles méridionales, et depuis douze ans chez les Ayorés du Paraguay (cf. Guerre et récit chez les indiens Ayorés du Chaco paraguayen). Spécialiste des questions de culture matérielle et de parenté spirituelle, a travaillé d'une part sur le rapport entre conditions matérielles d'existence et représentations, d'autre part sur la symbolique, les prohibitions et les possibilités matrimoniales déclenchées par les relations de parenté fictives, en élaborant les concepts d'atome de parenté spirituelle et d'inceste du troisième type (cf. L'esprit de la parenté); a organisé en 1976 en Sicile les premières expositions sur les outils de travail (cf. La mano di Prometeo, sur les arts $d u$ feu), ce qui lui a permis d'approfondir la réflexion sur les fondements matériels de la culture et de proposer les lignes d'une nouvelle muséographie anthropologique fondée sur la reconstitution, par phases d'avancement, des processus de travail (cf. La cultura materiale in linguistica e in antropologia). Il s'est consacré aux aspects rituels et symboliques des faits alimentaires (cf. Le ferment divin et Les tables de saint Joseph) et travaille actuellement sur l'allaitement et la symbolique du corps en Italie du sud. Il vient de publier aux éditions Les Belles Lettres Le sauvage et son double, 2011. 